\title{
Innate immunity receptors in depression and suicide: upregulated NOD-like receptors containing pyrin (NLRPs) and hyperactive inflammasomes in the postmortem brains of people who were depressed and died by suicide
}

\author{
Ghanshyam N. Pandey, PhD; Hui Zhang, PhD; Anuradha Sharma, PhD; Xinguo Ren, MD
}

\begin{abstract}
Background: Abnormalities of inflammation have been implicated in the pathophysiology of depression and suicide, based on observations of increased levels of proinflammatory cytokines in the serum of people who were depressed and died by suicide. More recently, abnormalities in cytokines and innate immunity receptors such as toll-like receptors have also been observed in the postmortem brains of people who were depressed and died by suicide. In addition to toll-like receptors, another subfamily of innate immunity receptors known as NOD-like receptors containing pyrin (NLRPs) are the most widely present NOD-like receptors in the central nervous system. NLRPs also form inflammasomes that play an important role in brain function. We studied the role of NLRPs in depression and suicide. Methods: We determined the protein and mRNA expression of NLRP1, NLRP3 and NLRP6 and the components of their inflammasomes (i.e., adaptor molecule apoptosis-associated speck-like protein [ASC], caspase1, caspase3, interleukin [IL]-1 $\beta$ and IL-18) postmortem in the prefrontal cortex of people who were depressed and died by suicide, and in healthy controls. We determined mRNA levels using quantitative polymerase chain reaction, and we determined protein expression using Western blot immunolabelling. Results: We found that the protein and mRNA expression levels of NLRP1, NLRP3, NLRP6, caspase3 and ASC were significantly increased in people who were depressed and died by suicide compared to healthy controls. Limitations: Some people who were depressed and died by suicide were taking antidepressant medication at the time of their death. Conclusion: Similar to toll-like receptors, NLRP and its inflammasomes were upregulated in people who were depressed and died by suicide compared to healthy controls. Innate immunity receptors in general - and NLRPs and inflammasomes in particular - may play an important role in the pathophysiology of depression and suicide.
\end{abstract}

\section{Introduction}

Suicide is a major public health concern. According to the National Center for Health Statistics (US Centers for Disease Control and Prevention), ${ }^{1} 48344$ suicides took place in 2018 in the United States alone. Many clinical, demographic and psychological studies of suicide have been conducted, but there are relatively few studies of the neurobiology of suicide. In recent years, it has become evident that abnormal immune function may be associated with suicide ${ }^{2}$ and depression., ${ }^{3,4}$ Cytokines are major mediators of inflammation (see reviews by Black and Miller ${ }^{2}$ and Dowlati and colleagues ${ }^{5}$ ); the hypothesis that abnormal immune function is associated with the pathogenesis of suicide ${ }^{6}$ and depression ${ }^{5}$ is based primarily on the observation that levels of proinflammatory cytokines such as interleukin (IL)-1 $\beta$, IL-6 and tumour necrosis factor (TNF) $\alpha$ are increased in the plasma, serum and cerebrospinal fluid of people who are depressed ${ }^{5}$ and suicidal. ${ }^{2}$ A role for cytokines in suicide and depression is also supported by the observations of Tonelli and colleagues, ${ }^{7}$ and by our observation ${ }^{8,9}$ that mRNA and protein levels of proinflammatory cytokines such as IL-1 $\beta$, IL-6 and TNF $\alpha$ were increased in the prefrontal cortex (PFC) of people who were depressed and died by suicide. However, the mechanism of increased cytokines in the postmortem brains of such people is unclear.

One of the mechanisms of cytokine production in the brain may be related to the activation of innate immune receptors. The innate immune system plays an important role in the central nervous system, especially in pathological conditions. ${ }^{4}$ In the brain, several cell types (such as microglia, astrocytes and neurons) express these innate immune receptors, ${ }^{10}$ which include subfamilies of toll-like receptors (TLRs) and NODlike receptors (NLRs; see review by Lampron and colleagues ${ }^{11}$ ). TLRs and NLRs are pattern-recognition receptors

Correspondence to: G.N. Pandey, University of Illinois at Chicago, 1601 West Taylor St., Chicago, IL 60612; gnpandey@ uic.edu

Submitted Jan. 19, 2021; Revised Apr. 7, 2021; Accepted Jun. 14, 2021

Cite as: J Psychiatry Neurosci 2021 September 29;46(5). doi: 10.1503/jpn.210016 
that recognize 2 types of ligands: pathogen-associated molecular patterns (PAMPs), linked to various microbes, and damage-associated molecular patterns (DAMPs), produced during tissue-based injury. ${ }^{12-14}$ After microbial recognition, both TLRs and NLRs activate signalling pathways that lead to the formation of cytokines and chemokines. ${ }^{15}$

PAMPs and DAMPs include proteins from bacterial membranes, intracellular proteins (such as heat-shock proteins) and protein products (such as adenosine triphosphate [ATP], urea and nucleic acid). ${ }^{16}$ In a previous study, we reported that the protein and mRNA expression of IL-1 $\beta$, IL- 6 and TNF $\alpha$ was increased in the PFC of adults ${ }^{9}$ and teens ${ }^{8}$ who were depressed and died by suicide. We have also found increased mRNA and protein expression of TLRs - particularly TLR2, TLR3, TLR4, TLR6 and TLR1017,18 — in the PFC of people who were depressed and died by suicide compared to healthy controls. This finding suggests that the increased IL-6 and TNF $\alpha$ we observed ${ }^{8,9}$ in the postmortem brains of people who were depressed and died by suicide may have been related to increases in specific TLRs. However, the formation of IL-1 $\beta$ involves a 2-step process: the activation of TLRs leads to increased transcription of pro-IL-1 $\beta$, which is then processed by the activation of NOD-like receptors containing pyrin (NLRP) inflammasomes. ${ }^{19}$ In general, the inflammasomes consist of an NLRP, the adaptor molecule apoptosis-associated speck-like protein (ASC), a protein containing a caspase recruitment domain and the downstream effector enzyme pro-caspase $1 .^{20,21}$

Because the conversion of pro-IL-1 $\beta$ to active or mature IL-1 $\beta$ involves the activation of NLRP inflammasomes, our objective in this study was to examine whether increased protein and mRNA expression of IL-1 $\beta$ in the postmortem brains of people who were depressed and died by suicide ${ }^{8,9}$ was related to abnormalities in the components of NLRP inflammasomes.

The NLR family has 21 members; of these, NLRP1, NLRP3 and NLRP6 show the highest levels of expression in the central nervous system, mainly in microglia, but also in astrocytes and neurons, ${ }^{22}$ and they form inflammasomes. To examine whether increased IL- $1 \beta$ in people who were depressed and died by suicide was related to increased activity of NLRP inflammasomes, we determined the mRNA and protein expression of the following in the PFC of 24 people who were depressed and died by suicide and 24 healthy controls: NLRP1, NLRP3 and NLRP6; the components of NLRP3 inflammasomes (i.e., ASC and caspase1); and the components of the product of NLRP3 inflammasome activation (i.e., IL-1 $\beta$ and IL-18).

\section{Methods}

\section{Participants and diagnoses}

This study was performed in the PFC (Brodmann area 9) of 24 people who were depressed and died by suicide and 24 nonpsychiatric controls (healthy controls). Brain tissues were obtained from the Maryland Brain Collection at the Maryland Psychiatric Research Center in Baltimore. Tissues were collected only after a family member had provided informed consent. All tissue from healthy controls and people who were depressed and died by suicide was grossly examined by experienced neuropathologists. Toxicology data were obtained by analysis of urine and blood samples. All procedures were approved by the institutional review boards of the University of Maryland and University of Illinois at Chicago.

\section{Diagnostic method}

Diagnoses were based on the Structured Clinical Interview for DSM-IV (SCID I). ${ }^{23}$ At least 1 family member or a friend underwent an interview after providing written informed consent. Two psychiatrists made diagnoses by consensus based on data obtained in the interview, medical records from the case and records obtained from the medical examiner's office. We verified that healthy controls lacked mental illnesses using the same consensus diagnostic procedures.

\section{Determination of $m R N A$ levels}

Total RNA from $100 \mathrm{mg}$ of frozen tissue was extracted using Trizol reagent (Invitrogen; Thermo Fisher Scientific Inc.), followed by DNase treatment according to the manufacturer's protocol. Concentration of total RNA samples was measured using a NanoDrop 1000 (NanoDrop Technologies, LLC), and purity was determined as a $260 / 280 \mathrm{~nm}$ ratio of 1.8 to 2.0. The quality of the RNA was assessed using a 2100 Bioanalyzer (Agilent Technologies, Inc.) to determine the RNA integrity number (RIN). All samples had 28S/18S ratios greater than 1.2 and RINs greater than 7.0. Finally, RNA samples were stored at $-80^{\circ} \mathrm{C}$ until required for further analysis.

The mRNA expression levels were determined using a 2-step, real-time, quantitative polymerase chain reaction (qPCR) method as follows: reverse transcription in duplicate $\left(25^{\circ} \mathrm{C}, 10\right.$ minutes; $37^{\circ} \mathrm{C}, 60$ minutes; $70^{\circ} \mathrm{C}, 15$ minutes; then hold at $4^{\circ} \mathrm{C}$ ); qPCR in duplicate from each reverse transcription $\left(95^{\circ} \mathrm{C}, 10\right.$ minutes; then $95^{\circ} \mathrm{C}$ for 15 seconds and $60^{\circ} \mathrm{C}$ for 30 seconds, cycled 40 times). For reverse transcription, $1 \mu \mathrm{g}$ of total RNA was reverse-transcribed using $50 \mathrm{ng}$ of random hexamers, $2 \mathrm{mM}$ dNTP mix, 10 units of ribonuclease inhibitor and 200 units of MMLV reverse transcriptase enzyme in a final reaction volume of $20 \mu \mathrm{L}$. Real-time qPCR was performed using predesigned TaqMan gene expression assays (Applied Biosystems; Thermo Fisher Scientific Inc.) for all target and housekeeping genes on a MX3005p sequence detection system (Agilent Technologies, Inc.). The TaqMan assay IDs for the housekeeping and target genes are listed in Table 1.

To determine the stability and optimal number of the housekeeping genes, we used geNORM version 3.4 (PrimerDesign Ltd.) according to the manufacturer's instructions. The average gene-stability measure ranked $\beta$-actin and glyceraldehyde 3-phosphate dehydrogenase (GAPDH) as the most stable genes in our samples. We tested qPCR efficiency over a 5-log dilution series and confirmed that all target genes and housekeeping genes had similar amplification efficiencies. For each primer-probe set, we performed qPCR using $10 \mu \mathrm{L}$ of cDNA diluted 1:10-fold. Each qPCR plate included "no reverse transcriptase" and "no template" controls 
Table 1: Primers/probes used for real-time PCR analysis

\begin{tabular}{lccc}
\hline Primer/probe & TaqMan accession & $\begin{array}{c}\text { Probe location } \\
\text { (exon boundary) }\end{array}$ & Assay function \\
\hline ACTB & Hs99999903_m1 & $1-1$ & Housekeeping gene \\
GAPDH & Hs99999905_m1 & $3-3$ & Housekeeping gene \\
NLRP1 & Hs00248187_m1 & $6-7$ & Target gene \\
NLRP3 & Hs00918082_m1 & $1-2$ & Target gene \\
NLRP6 & Hs00373246_m1 & $5-6$ & Target gene \\
CASP1 & Hs00354836_m1 & $8-9$ & Target gene \\
CASP3 & Hs00234387_m1 & $4-5$ & Target gene \\
PYCARD/ASC & Hs00203118_m1 & $2-3$ & Target gene \\
IL18 & Hs01038788_m1 & $3-4$ & Target gene \\
\hline \multicolumn{2}{l}{ PCR = polymerase chain reaction. } & & \\
\hline
\end{tabular}

to eliminate nonspecific amplification. One sample from each target gene was run on a gel to confirm specificity, and all samples were run in triplicate. Target gene qPCR data were normalized to the geometric mean of $\beta$-actin and GAPDH and were expressed relative to the control samples using the $2^{-\Delta \Delta \mathrm{Ct}}$ method.

\section{Determination of protein levels}

The cytosol fraction of the brain tissue samples was prepared by homogenizing $100 \mathrm{mg}$ of tissue in $0.6 \mathrm{~mL}$ of buffer containing $100 \mathrm{mM}$ Tris (pH 7.4); $150 \mathrm{mM} \mathrm{NaCl} ; 1 \%$ Triton $\mathrm{X}-100 ; 1 \%$ sodium decanoate; $0.1 \%$ sodium dodecyl sulfate; $5 \mathrm{mM}$ ethylenediaminetetraacetic acid; $1 \mathrm{mM}$ phenylmethylsulfonyl fluoride; $5 \mu \mathrm{g} / \mathrm{mL}$ aprotinin, leupeptin and pepstatin; and $100 \mathrm{mM}$ sodium orthovanadate. The homogenate was centrifuged at $15000 \mathrm{~g}$ for 10 minutes at $4^{\circ} \mathrm{C}$, and the supernatant was used for all assays. Protein concentration was determined according to the method of Lowry and colleagues. ${ }^{24}$

\section{Quantitation of NLRPS, ASC, caspase1 and caspase3}

Immunolabelling of NLRP1, NLRP3, NLRP6, caspase1, caspase 3 and ASC was performed as described previously. ${ }^{25}$ Briefly, $20 \mu \mathrm{g}$ protein samples were loaded onto 7.5\% (wt./ vol.) acrylamide gel. The proteins were then transferred electrophoretically to an enhanced chemiluminescent nitrocellulose membrane. Blots were incubated with anti-NLRP1, NLRP3, NLRP6, caspase1, caspase3 and ASC antibodies overnight and with horseradish peroxidase-linked secondary antibody for 3 hours at room temperature, exposed to enhanced chemiluminescent film. Membranes were probed by monoclonal $\beta$-actin antibody (1:5000 for 2 hours), followed by secondary anti-mouse IgG antibody (1:5000 for 2 hours). The bands on the autoradiogram were quantified using the Loats Image Analysis System (Loats Associates, Inc.). The optical density of each band for NLRP1, NLRP3, NLRP6, caspase1, caspase 3 and ASC was corrected using the optical density of the corresponding $\beta$-actin band. Values were presented as a percentage of the control. The NLRP1, NLRP3, caspase1, caspase 3 and ASC polyclonal antibodies were purchased from
Cell Signalling Technology, Inc.; the NLRP6 antibody was purchased from Abcam.

\section{Determination of IL-18 protein levels}

Levels of IL-18 were determined in aliquots of the cytosol fraction by enzyme-linked immunosorbent assay using commercially available Quantikine kits for human IL-18, purchased from R \& D Systems.

\section{Statistical analysis}

We performed data analyses using SPSS statistical software (version 27; IBM). All data were embedded in a generalized linear model to compare the effects of the 2 groups (people who were depressed and died by suicide and healthy controls) on the mRNA and protein expression of NLRP and NLRP-associated inflammasome molecules by adjusting the effects of age, sex, postmortem interval and brain $\mathrm{pH}$. To examine whether the protein and mRNA expression of NLRPs and other outcome measures were affected by postmortem interval, age, or brain $\mathrm{pH}$, we included these variables as covariates in the multivariate model. We analyzed the results for protein and mRNA expression individually using SPSS. We also tested each outcome measure for normality using the Kolmogorov-Smirnov test. We performed univariate comparisons between the 2 groups for each studied molecule using the Bonferroni post hoc test. Changes with $p$ values of 0.05 or less were considered significant.

\section{Results}

\section{Sample demographic and clinical characteristics}

The demographic and clinical characteristics of the 2 study groups are summarized in Table 2, and detailed characteristics are provided in Appendix 1, Table S1, available at jpn.ca. This report describes observations for variables such as age, sex, race or ethnicity, postmortem interval and brain $\mathrm{pH}$; Appendix 1, Table S1, also includes the method of suicide and the presence of antidepressants or ethanol at the time of death for people who were depressed and died by suicide. 
Table 2: Participant demographic and clinical characteristics

\begin{tabular}{lcc} 
Characteristic & Healthy controls* & $\begin{array}{c}\text { Depressed and } \\
\text { died by suicide }\end{array}$ \\
\hline Participants, $n$ & 24 & 24 \\
Age, yr & $42.08 \pm 15.35$ & $38.95 \pm 15.39$ \\
Race or ethnicity & 7 Black, 17 White & 2 Black, 22 White \\
Sex & 20 male, 4 female & 14 male, 10 female \\
Postmortem interval, h & $16.54 \pm 6.4$ & $18.91 \pm 6.02$ \\
Brain pH & $7.01 \pm 0.15$ & $6.96 \pm 0.25$ \\
\hline
\end{tabular}

*Values are presented as $n$ or mean \pm standard deviation.

\section{Protein and $m R N A$ expression of IL-1 $\beta, I L-6$ and TNF $\alpha$}

In an earlier study, we determined the protein and mRNA expression of TNF $\alpha$, IL-1 $\beta$ and IL- 6 in the PFC of healthy controls and people who were depressed and died by suicide. ${ }^{9}$ Compared to healthy controls, we found a significant increase in the protein and mRNA expression of TNF $\alpha$, IL-1 $\beta$ and IL- 6 in the PFC of people who were depressed and died by suicide (Figure $1 \mathrm{~A}$ and $\mathrm{B}$ ).

\section{mRNA expression}

We determined the mRNA expression of 3 NLRPs (NLRP1, NLRP3 and NLRP6) using qPCR, followed by mRNA expression studies of some component genes of NLRP3-associated inflammasomes (ASC, caspase1, caspase3 and IL-18); results are shown as fold change (Figure $2 \mathrm{~A}$ and $\mathrm{B}$ ). Comparison of the mRNA expression of these genes in both groups using multivariate analysis showed overall significant differences in the mRNA expression of NLRP3 $\left(F_{4,50}=1.331 ; p=0.018\right)$ and caspase $3\left(F_{4,50}=2.654 ; p=0.045\right)$. Univariate comparisons using the Bonferroni post hoc test suggested significant upregulation of the mRNA expression of NLRP1 $\left(F_{1,45}=5.127\right.$; $p=0.028), \operatorname{NLRP3}\left(F_{1,45}=4.656 ; p=0.036\right)$ and $\operatorname{NLRP6}\left(F_{1,45}=\right.$ $4.944 ; p=0.031)$ in the PFC of people who were depressed and died by suicide compared to healthy controls (Figure 2A). Univariate comparison tests also revealed significant increases in the mRNA expression of $\operatorname{ASC}\left(F_{1,45}=4.377 ; p=\right.$ $0.042)$ and caspase $3\left(F_{1,45}=6.187 ; p=0.017\right)$ in the PFC of people who were depressed and died by suicide compared to healthy controls (Figure 2B). However, we observed no significant differences between the 2 groups in the mRNA expression of caspase1 and IL-18 (Figure 2B). Confounding variables such as age, postmortem interval and brain $\mathrm{pH}$ showed no significant effects on mRNA expression in either of the 2 study groups.

To examine whether mRNA expression results had a normal bimodal distribution, we performed the KolmogorovSmirnov test of normality in SPSS and observed that mRNA expression of NLRP1 in people who were depressed and died by suicide failed the normality test $(p \leq 0.05)$. NLRP1 in healthy controls and other measures in both groups passed the normality test.

\section{Protein expression}

We also studied the protein expression of NLRPs and NLRP3associated inflammasome protein components using Western blotting; the results are shown as representative blots and histograms in Figure 3 and Figure 4. Statistical analysis of protein levels using multivariate analysis showed significant overall changes in the protein expression of NLRP1 $\left(F_{4,49}=3.586, p=\right.$ 0.013), NLRP3 $\left(F_{4,49}=3.405 ; p=0.016\right), \operatorname{NLRP6}\left(F_{4,49}=3.358 ; p=\right.$ 0.018; Figure 3B) and ASC $\left(F_{4,49}=4.987, p=0.002\right.$; Figure $\left.4 \mathrm{~B}\right)$ in the PFC of people who were depressed and died by suicide compared to healthy controls. Univariate comparison using the Bonferroni post hoc test showed significant increases in the protein expression of NLRP1 $\left(F_{1,44}=13.573 ; p=0.001\right)$, NLRP3 $\left(F_{1,44}=12.164 ; p=0.001\right)$ and NLRP6 $\left(F_{1,44}=5.193 ; p=\right.$ $0.028)$ in people who were depressed and died by suicide (Figure 3B). Protein expression of ASC $\left(F_{1,44}=18.796 ; p<0.001\right)$ and caspase $3\left(F_{1,44}=3.987 ; p=0.05\right.$; Figure 4$)$ were also
A

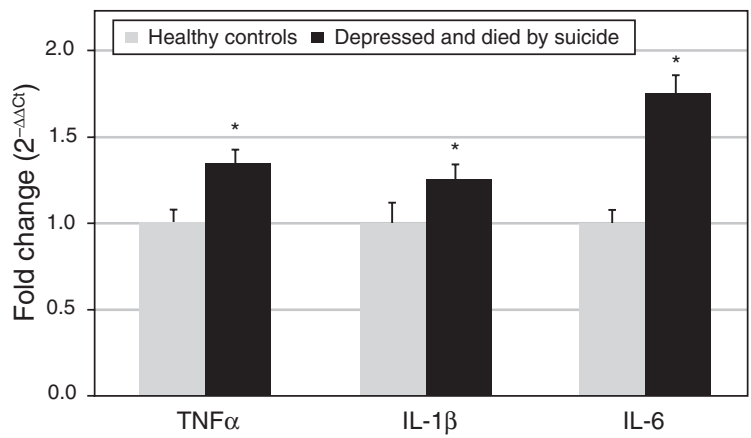

B

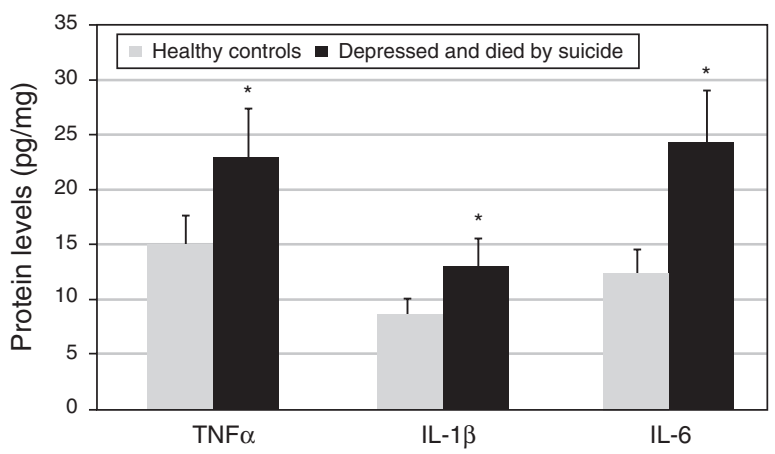

Figure 1: (A) Mean mRNA expression levels of TNF $\alpha, I L-1 \beta$ and IL- 6 in the prefrontal cortex of healthy controls and people who were depressed and died by suicide. The data are shown as the fold change in mRNA levels \pm standard error of the mean. (B) Mean protein expression levels (pg/mg tissue) of TNF $\alpha$, IL-1 $\beta$ and IL- 6 in the prefrontal cortex of healthy controls and people who were depressed and died by suicide. Values are given as the mean \pm standard deviation; ${ }^{*} p<0.05$. IL $=$ interleukin; TNF $\alpha=$ tumour necrosis factor- $\alpha$. 
A

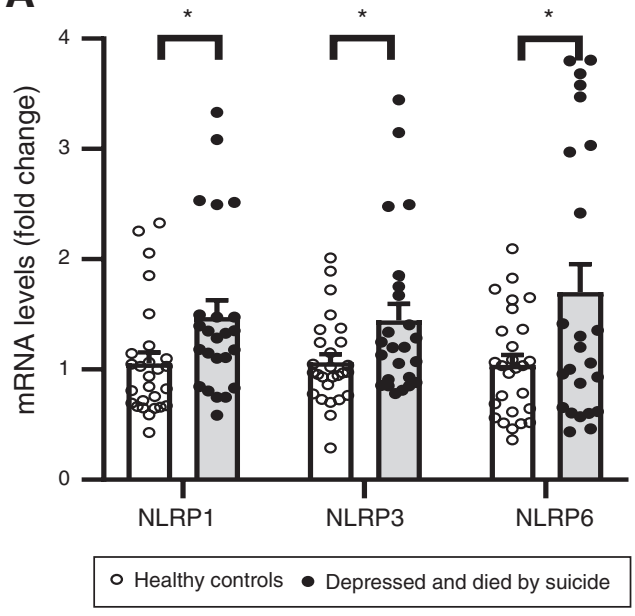

B

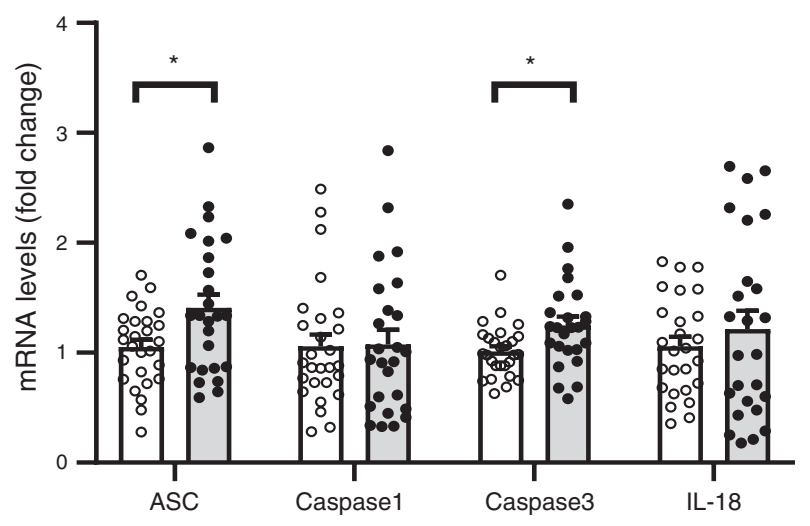

- Healthy controls - Depressed and died by suicide

Figure 2: (A) mRNA expression of NLRPs. Histograms represent fold change in the mRNA expression of NLRP1, NLRP3 and NLRP6 in the prefrontal cortex of healthy controls and people who were depressed and died by suicide. Values represent mean fold change \pm standard error of the mean; ${ }^{*} p \leq 0.05$. (B) mRNA expression of genes associated with the NLRP3 inflammasome. Histograms represent fold change in the mRNA expression of ASC, caspase1, caspase3 and IL-18 in the prefrontal cortex of healthy controls and people who were depressed and died by suicide. Values represent mean fold change \pm standard error of the mean; ${ }^{*} p \leq 0.05$. ASC $=$ apoptosis-associated speck-like protein; $\mathrm{IL}=$ interleukin; NLRP = NOD-like receptor containing pyrin.

A

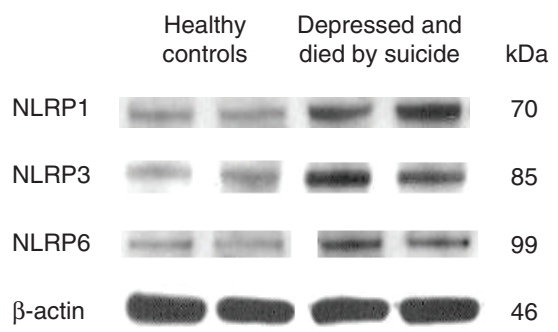

B

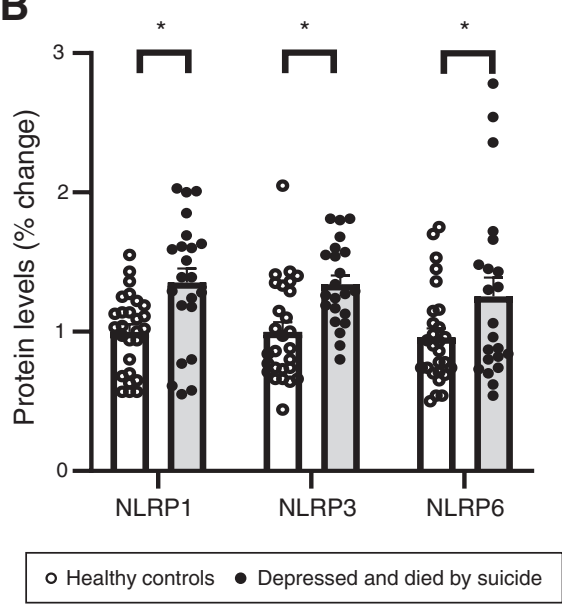

Figure 3: Protein expression of NLRPs. (A) Representative blots for 3 different NLRPs (NLRP1, NLRP3 and NLRP6) and (B) histograms showing corresponding percent change in protein expression of these NLRPs in the prefrontal cortex of healthy controls and people who were depressed and died by suicide. Values represent mean percent change \pm standard error of the mean; ${ }^{*} p \leq 0.05$. NLRP $=$ NOD-like receptor containing pyrin.

upregulated significantly in the PFC of people who were depressed and died by suicide, as suggested by representative blots (Figure 4A) and pair-wise comparison testing (Figure 4B). Protein expression of caspase1 (Figure 4B) and IL-18 (Figure 4C) showed no significant differences between the 2 study groups. These results were similar to and consis- tent with our mRNA results (Figure 2B). We found no significant effects of confounding variables (age, postmortem interval or brain $\mathrm{pH}$ ) on protein expression in either study group, except for an effect of age on the protein expression of IL-18 $(p=0.045)$ and an effect of postmortem interval on the protein expression of NLRP6 $(p=0.046)$. 


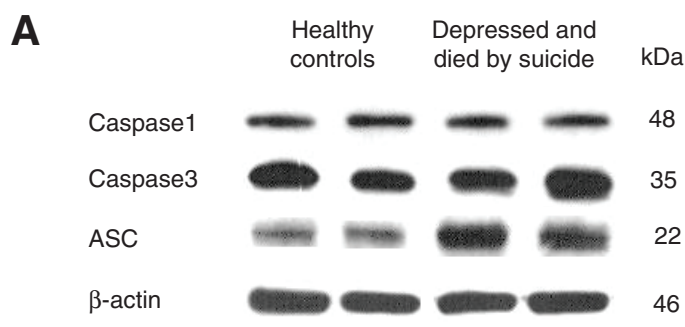

B

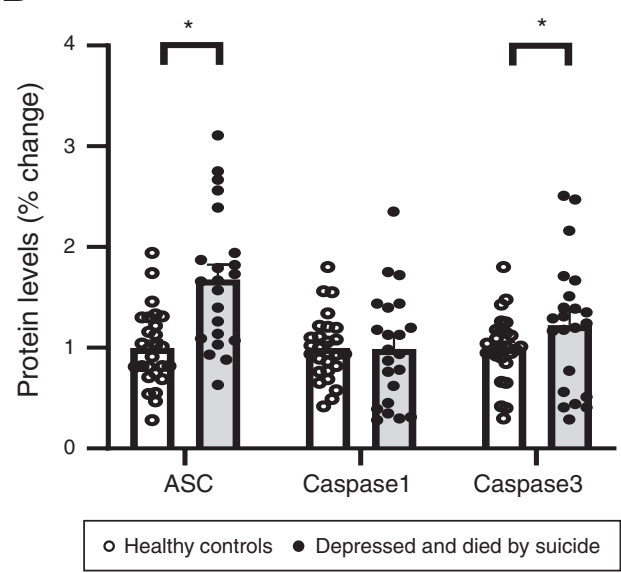

C

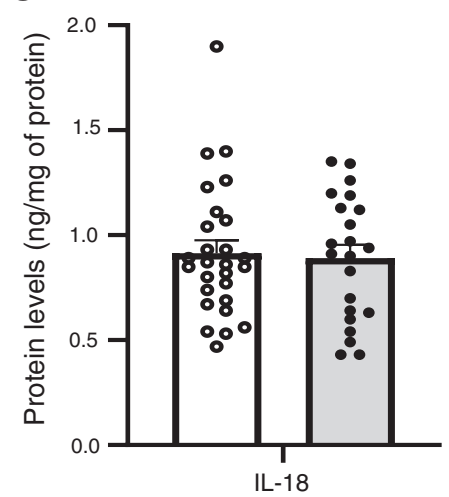

- Healthy controls - Depressed and died by suicide

Figure 4: Protein expression of molecules associated with the NLRP3 inflammasome. (A) Representative blots for ASC, caspase1 and caspase3. (B) Histograms showing corresponding percent change in protein expression of these inflammasome members in the prefrontal cortex of healthy controls and people who were depressed and died by suicide. (C) Histograms showing protein expression of IL-18 in the prefrontal cortex of healthy controls and people who were depressed and died by suicide (measured by enzyme-linked immunosorbent assay. Values represent mean percent change \pm standard error of the mean; ${ }^{*} p \leq 0.05$. ASC $=$ apoptosis-associated speck-like protein; $\mathrm{IL}=$ interleukin; NLRP $=$ NOD-like receptor containing pyrin .

\section{Effect of suicide method and antidepressants on $m R N A$ and protein expression}

Because some studies have reported that suicide method is an important variable, we determined the effect of suicide method (i.e., gunshot wound, hanging or drug overdose) on our variables using SPSS.

In multivariate analysis, suicide method had no significant effect on the mRNA or protein expression of any of the molecular markers studied. We also determined the effect of 3 suicide methods (gunshot wound, hanging and drug overdose) separately for every marker in those who were depressed and died by suicide, and we observed no significant effects $(p \geq 0.45)$.

We also examined whether changes in the protein and mRNA expression of NLRPs and NLRP-associated inflammasome molecules that we observed in the PFC of people who were depressed and died by suicide were related to previous treatment, the presence of antidepressant drugs or both. Among those who were depressed and died by suicide 11 of 24 were taking antidepressants at the time of their death. We divided this group into 2 subgroups (those taking antidepressants and those not taking antidepressants) and performed $t$ tests. We found no significant differences in the means of the 2 subgroups for mRNA expression (except NLRP1, $p \leq 0.01$ ) or protein expression, suggesting that apart from NLRP1 mRNA expression, antidepressants had no significant effect on the gene or protein expression of NLRPs and NLRP-associated inflammasome molecules.

\section{Discussion}

In the present study we found that the mRNA and protein expression of NLRP1, NLRP3, NLRP6 and ASC were significantly increased in the PFC of people who were depressed and died by suicide compared to healthy controls. However, we found no significant differences between the 2 groups in the expression of caspase 1 or IL-18.

Because caspase 1 activation increases IL-18, and caspase3 inactivates IL- 18 but not IL- $1 \beta,{ }^{26}$ it is possible that our failure to find increased IL-18 may have been because of the increased caspase 3 we observed. In a previous report, ${ }^{9}$ we found that the mRNA and protein expression of IL-1 $\beta$ was significantly increased in people who were depressed and 
died by suicide compared to healthy controls. We did not find significant changes in the expression of IL-18 between the 2 groups, suggesting that IL-18 abnormality is not involved in depression and suicide.

The NLRs comprise 3 different domains: the C-terminal domain, which consists of leucine-rich repeats and is thought to recognize microglial PAMPs and DAMPs or endogenous host molecules; a conserved central nucleotide binding and oligomerization domain (NOD or NACHT) and an N-terminal pyrin domain; ${ }^{27}$ and a pyrine domain, which is required for its activation. ${ }^{19,28}$ A unique feature of the NLRPs in general and NLRP3 in particular is that they are involved in forming a multiprotein complex known as an inflammasome..$^{20,29}$ The NLRP inflammasome consists primarily of a cytosolic sensor molecule NLRP3 or other NLRPs, the adaptor protein ASC and a cysteine protease pro-caspase as the effector molecule. ${ }^{21}$ It is formed when NLRP3 associates with the adaptor protein ASC and pro-caspase1, 19,30 upon activation by different stimuli, such as PAMPs or DAMPs. The activation of inflammasomes results in the cleavage of caspase1 from its pro-form to its enzymatically active form. The activated caspase 1 then causes the cleavage of its several substrates, such as pro-IL-1 $\beta$ and pro-IL-18 to active IL-1 $\beta$ and IL-18. ${ }^{29}$

Although all 3 of the NLRPs we studied form inflammasomes, the NLRP3 inflammasome is the most widely studied at both the basic and clinical levels. ${ }^{19,31}$ It has also been studied in neurodegenerative disorders such as Alzheimer disease. $^{31-33}$ To the best of our knowledge, neither the NLRP innate immunity receptors nor the NLRP inflammasomes have been studied in the brains of people who were depressed or died by suicide. However, the NLRP3 receptor and its inflammasomes have been studied in the postmortem brains of people with bipolar disorder by Kim and colleagues, ${ }^{34}$ who found higher mRNA levels of mitochondrial NLRP3 and ASC in people with bipolar disorder. They also found increased levels of IL-1 $\beta, \mathrm{TNF} \alpha$ and caspase 1 in the postmortem brains of people with bipolar disorder and schizophrenia.

Alcocer-Gomez and colleagues ${ }^{35}$ found higher mRNA expression of NLRP3 and ASC in the peripheral blood mononuclear cells and higher protein expression of IL-1 $\beta$ and IL-18 in the serum of patients with major depressive disorder compared to controls. Syed and colleagues ${ }^{36}$ evaluated the components of the NLRP3 inflammasome and found significantly increased levels of ASC, caspase1 and IL-18, but not IL-1 $\beta$, in the plasma of patients with major depressive disorder compared to healthy controls.

A role for the NLRP3 inflammasome in depression is also supported by stress-induced depressive behaviour in animal models of depression. ${ }^{37}$ Mice who were subjected to immobilization or the forced swim test exhibited increased levels of NLRP3 compared to control mice. As well, when stressed, NLRP3-knockout mice showed no depressive behaviour, microglial activation or reduced hippocampal neurogenesis. Thus, NLRP3 inhibition or deletion of microglial activation impairs the stress-induced alterations associated with depression. ${ }^{37}$
Another piece of evidence suggesting a role for the NLRP3 inflammasome in depression is derived from the studies of Pan and colleagues ${ }^{38}$ in rats treated with chronic unpredictable mild stress, a model of depression. The rats exhibited depressive-like behaviour, and the authors found increased protein and mRNA levels of NLRP3 and IL-1 $\beta$ in their PFC, suggesting a role for NLRP3 in depression. Thus, both clinical and animal studies suggest the involvement of NLRP inflammasomes in major depressive disorder.

The activation of NLRP3 is a 2-step process of priming and activation, as shown in Figure 5. Priming is achieved by recognition of PAMPs or DAMPs via TLRs or cytokines (such as TNF $\alpha$ or IL-1 $\beta$ ), or a combination of both. ${ }^{28,39}$ The second step consists of the activation of NLRPs by a wide variety of unrelated stimuli, including several DAMPs (such as ATP, urate or aluminum) and PAMPs (such as lipopolysaccharide, peptidoglycan or several toxins; see reviews by Swanson and colleagues ${ }^{28}$ and Vanaja and colleagues ${ }^{40}$ ).

Thus, there is distinct cooperation in the activation of NLRP3 inflammasomes between TLRs, cytokines and inflammasomes. The priming signal is provided by TLRs and cytokines such as TNF $\alpha$ with the formation of pro-IL-1 $\beta$ and proIL-18 (the substrates for the NLRP inflammasome), ${ }^{26}$ which are formed as a result of TLR interaction with PAMPs and DAMPs and the activation of nuclear factor $\kappa \mathrm{B}(\mathrm{NF}-\kappa \mathrm{B})$. Our studies showing abnormalities of specific TLRs in the PFC of people with depression who died by suicide ${ }^{17,18}$ confirmed that the priming signal for the activation of NLRP inflammasomes is increased, leading increased activation of NLRP inflammasomes themselves. As well, the main function of priming is to induce pro-IL-1 $\beta$ and potentiate NLRP3 inflammasome activity through NF- $\mathrm{KB}$-dependent induction of NLRP3, which may be a limiting component of the complex. ${ }^{41}$ Thus, it is primarily the upregulation of NLRPs (and probably ASC) that causes the IL- $1 \beta$ increase we have observed in the PFC of people who were depressed and died by suicide. ${ }^{9}$

Although the NLRP3 inflammasome has been more widely studied and characterized, NLRP1 and NLRP6 also form inflammasomes. In fact, NLRP1 inflammasomes were the first NLRP inflammasome to be characterized..$^{20}$ Although NLRP1 does not need ASC to form the inflammasome and can interact directly with pro-capsase 1 and bypass the ASC requirement, inclusion of ASC in the complex augments the activation of the human NLRP1 inflammasome. ${ }^{15,42}$ NLRP1 also interacts with caspase5, which may contribute to IL-1 $\beta$ processing in humans. ${ }^{20}$

NLRP6 forms an inflammasome as well, but the function of NLRP6 is not well known. This novel molecule was initially characterized as a regulator of NF- $\kappa \mathrm{B}$ and caspase $1 .{ }^{43}$ It functions as a positive activator of NF- $\kappa B$ when coexpressed with ASC, and it induces cytokine production. The increase in NLRP6 we found may not only cause the maturation of IL-1 $\beta$ but also lead to the production of other cytokines, such as TNF $\alpha^{15,42,43}$

ASC plays a central role in the activation of caspase 1 and the secretion of IL-1 $\beta$ and IL-18 in response to PAMPs and DAMPs. The activation of caspase 1 and the production of IL-1 $\beta$ 


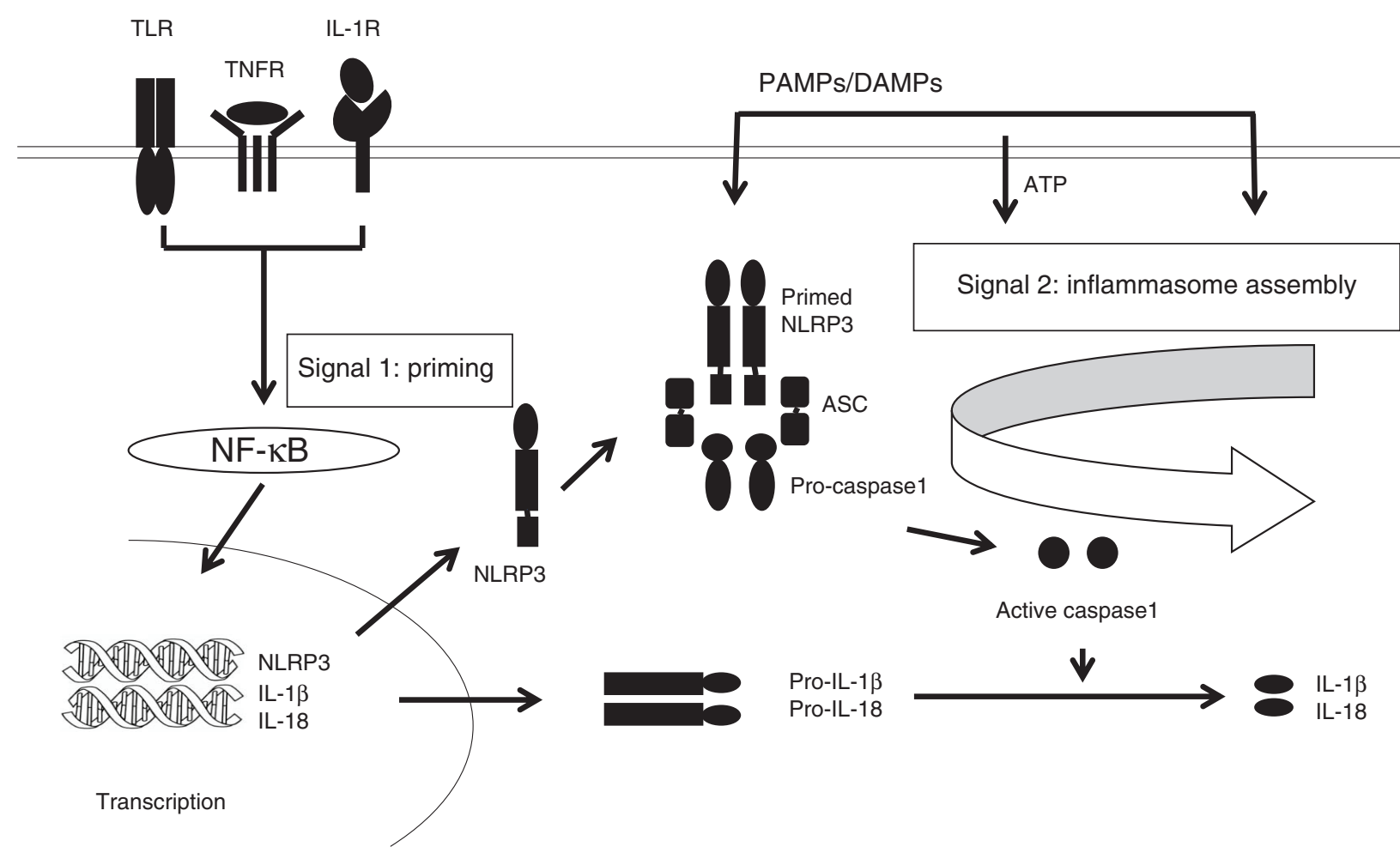

Figure 5: Activation of NLRP3 inflammasome and the formation of IL-1 $\beta$ and IL-18. Two signals are required for the activation of the NLRP3 inflammasome and the formation of IL-1 $\beta$ and IL-18. Signal 1 engages pattern-recognition receptors (such as TLRs, TNFRs or IL-1Rs) and activates NF-KB, leading to the transcription and translation of pro-IL-1 $\beta$ and pro-IL-18. Signal 2 is mediated by stimulation of PAMPs or DAMPs and promotes the assembly of the NLRP3 inflammasome complex. NLRP3 is activated by a wide variety of signals, including ATP, uric acid and aluminum. Upon activation, NLRP3 recruits pro-caspase1, which is subsequently changed into active caspase1. Active caspase1 then cleaves the pro-forms of IL-1 $\beta$ and IL-18 into their active forms and eventually induces cell death by pyroptosis. ATP = adenosine triphosphate; DAMP = damage-associated molecular pattern; IL = interleukin; IL-1R = IL-1 receptor; NF- $\mathrm{KB}=$ nuclear factor $\kappa B$; NLRP = NOD-like receptor containing pyrin; PAMP = pathogen-associated molecular patterns; TLR = toll-like receptor; TNFR = tumour necrosis factor receptor

and IL-18 is abolished in ASC-deficient macrophages, ${ }^{44}$ showing a crucial role for ASC in the IL-1 $\beta$ and IL-18 formation.

The other question is the cause and consequences of NLRP3 inflammasomes. The main causes of NLRP3 inflammasomes may be related to environmental factors, such as exposure to PAMPs and DAMPs. Exposure of TLRs to PAMP ligands, such as lipopolysaccharides produced by pathogens or mitochondria, ${ }^{45,46}$ may be a factor in activating NLRPs (see review by Vanaja and colleagues ${ }^{40}$ ). The generation of ATP through DAMPs, as well as exposure to toxic materials such as potassium ATP or mitochondrial-derived reactive oxygen species ${ }^{47}$ or uric acid ${ }^{48-50}$ may also be factors involved in the activation of NLRP3. ${ }^{20,50}$ Increased levels of reactive oxygen species and mitochondrial dysfunction have also been found in the postmortem brains of people with bipolar disorder, ${ }^{46,51,52}$ another factor for the upregulation of NLRP inflammasomes.

Inflammasome activation has behavioural, clinical and biological consequences. ${ }^{53}$ For example, active inflammasomes and increased IL-1 $\beta$ have been associated with a loss of cognitive function..$^{54,55}$ NLRP3 or caspase1 deficiency substantially attenuates the impairment of spatial memory. ${ }^{31,56}$ In addition to the behavioural abnormalities caused by NLRP inflammasomes and IL-1 $\beta$, the latter also causes many biological effects. IL-1 $\beta$ plays an important role in the initiation and continuation of the inflammatory reaction in the central nervous system in response to various adverse stimuli. It also reduces the integrity of the blood-brain barrier, allowing the infiltration of peripheral immune cells into the central nervous system. ${ }^{11}$

However, one of the specific effects of IL- $1 \beta$ is an increase in a process known as pyroptosis. ${ }^{19,57}$ Increased levels of caspase1 activated by the NLRP3 inflammasome can result in a highly inflammatory form of cell death known as pyroptosis..$^{20,57}$ Pyroptosis occurs most frequently after infection with intracellular pathogens. ${ }^{58}$ The process of pyroptosis is exclusively mediated by caspase1, is different from apoptosis and occurs independently of proapoptotic caspases. ${ }^{57}$ The major consequences of increased inflammasome activity that we observed in people who were depressed and died by suicide may have been increased cell death mediated by pyroptosis in the brain. 
Abnormalities in IL-1 $\beta$, IL-18 and NLRP3 inflammasomes have been observed in neurodegenerative disorders such as Alzheimer disease. A recent report showed that protein and mRNA levels of IL-18 were significantly increased in the postmortem brains of people with Alzheimer disease, especially in the frontal lobe. ${ }^{59}$ Heneka and colleagues ${ }^{56}$ reported that expression of NLRP3 and caspase1 was significantly increased in the postmortem brains of people with Alzheimer disease.

The observation that NLRP3 inflammasomes and IL-1 $\beta$ in were increased the brains of people who were depressed and died by suicide provides a potential therapeutic target. NLRP3 may be a unique target in several ways. Abnormalities in cytokines and TLRs enhance the activation of NLRP3. Some studies have identified compounds that may block IL-1 signalling or cause the inhibition of NLRP3 inflammasome activation. ${ }^{28,60}$ Several compounds related to the IL-1 receptor and NLRP3 are being tested in conditions such as inflammatory and neurodegenerative disorders. ${ }^{31}$

\section{Limitations}

This study had 2 main limitations. We did not have information about body mass index for the people in our study groups, and we were not certain if any of the observed changes in NLRP and its inflammasomes were related, at least in part, to body mass index. Some people were taking antidepressant treatment at the time of their death. Although we did not observe differences in the gene and protein expression of NLRPs and NLRP-associated inflammasome molecules between those who were taking antidepressants and those who were not, this is one of the limitations of a postmortem brain study.

\section{Conclusion}

The innate immunity system in the brain is mediated by subfamilies of innate immunity receptors such as TLRs and NLRPs - also known as pattern recognition receptors which recognize PAMPs and DAMPs. Interaction of PAMPs and DAMPs with these receptors initiates a signalling mechanism, resulting in the activation of NF- $\mathrm{KB}$ and the production of cytokines such as pro-IL-1, pro-IL-18, IL-6 cytokines and other chemokines. Our studies have indicated increased NLRP1, NLRP3, NLRP6 and IL-1 $\beta$ signalling; the upregulation of NLRP1, NLRP3 and NLRP6 in general; and the upregulation of inflammasomes, consisting of ASC and caspase1 in particular. Activation of this multiprotein complex by PAMPs and DAMPs causes the activation and cleavage of caspase1; active caspase 1 in turn cleaves pro-IL-1 $\beta$ and proIL-18, resulting in the formation of IL-1 $\beta$ and IL-18, which perform many important functions in the central nervous system, including pyroptosis (programmed cell death, which is different than apoptosis). These results not only provide a mechanism for the involvement of NLRPs in the pathophysiology of depression and suicide, but they also show great potential for developing specific therapeutics to treat depression and suicidal behaviour, especially in those who are unresponsive to antidepressants.
Affiliations: From the Department of Psychiatry, University of Illinois at Chicago Ringgold Standard Institution, Chicago, Illinois.

Funding: This research was supported by grants RO1MH098554 and RO1MH106565 (G. Pandey) from the National Institute of Mental Health, Rockville, Maryland. The funding source had no role in the study design; collection, analysis and interpretation of data, or in the writing of the manuscript.

Competing interests: None declared.

Contributors: G. Pandey designed the study. H. Zhang and $X$. Ren acquired the data, which A. Sharma analyzed. G. Pandey wrote the article, which $\mathrm{H}$. Zhang, A. Sharma and X. Ren reviewed. All authors approved the final version to be published and can certify that no other individuals not listed as authors have made substantial contributions to the paper.

Content licence: This is an Open Access article distributed in accordance with the terms of the Creative Commons Attribution (CC BY-NC-ND 4.0) licence, which permits use, distribution and reproduction in any medium, provided that the original publication is properly cited, the use is noncommercial (i.e., research or educational use), and no modifications or adaptations are made. See: https://creativecommons.org/licenses/by-nc-nd/4.0/

\section{References}

1. Xu J, Murphy SL, Kochanek KD, et al. Mortality in the United States, 2018. NCHS Data Brief no. 355. Hyattsville (MD): National Center for Health Statistics; 2020.

2. Black C, Miller BJ. Meta-analysis of cytokines and chemokines in suicidality: distinguishing suicidal versus nonsuicidal patients. Biol Psychiatry 2015;78:28-37.

3. Schiepers OJ, Wichers MC, Maes M. Cytokines and major depression. Prog Neuropsychopharmacol Biol Psychiatry 2005;29:201-17.

4. Wohleb ES, Franklin T, Iwata M, et al. Integrating neuroimmune systems in the neurobiology of depression. Nat Rev Neurosci 2016;17:497-511.

5. Dowlati Y, Herrmann N, Swardfager W, et al. A meta-analysis of cytokines in major depression. Biol Psychiatry 2010;67:446-57.

6. Serafini G, Pompili M, Elena Seretti M, et al. The role of inflammatory cytokines in suicidal behavior: a systematic review. Eur Neuropsychopharmacol 2013;23:1672-86.

7. Tonelli LH, Stiller J, Rujescu D, et al. Elevated cytokine expression in the orbitofrontal cortex of victims of suicide. Acta Psychiatr Scand 2008;117:198-206.

8. Pandey GN, Rizavi HS, Ren X, et al. Proinflammatory cytokines in the prefrontal cortex of teenage suicide victims. J Psychiatr Res 2012;46:57-63.

9. Pandey GN, Rizavi HS, Zhang H, et al. Abnormal protein and mRNA expression of inflammatory cytokines in the prefrontal cortex of depressed individuals who died by suicide. J Psychiatry Neurosci 2018;43:376-85.

10. Rosenzweig HL, Planck SR, Rosenbaum JT. NLRs in immune privileged sites. Curr Opin Pharmacol 2011;11:423-8.

11. Lampron A, Elali A, Rivest S. Innate immunity in the CNS: redefining the relationship between the CNS and its environment. Neuron 2013;78:214-32.

12. Franchi L, Eigenbrod T, Munoz-Planillo R, et al. The inflammasome: a caspase-1-activation platform that regulates immune responses and disease pathogenesis. Nat Immunol 2009;10:241-7.

13. Ye Z, Ting JP. NLR, the nucleotide-binding domain leucine-rich repeat containing gene family. Curr Opin Immunol 2008;20:3-9.

14. Takeuchi O, Akira S. Pattern recognition receptors and inflammation. Cell 2010;140:805-20.

15. Rathinam VA, Vanaja SK, Fitzgerald KA. Regulation of inflammasome signaling. Nat Immunol 2012;13:333-42.

16. Kumar H, Kawai T, Akira S. Pathogen recognition by the innate immune system. Int Rev Immunol 2011;30:16-34.

17. Pandey GN, Rizavi HS, Bhaumik R, et al. Innate immunity in the postmortem brain of depressed and suicide subjects: role of toll-like receptors. Brain Behav Immun 2019;75:101-11.

18. Pandey GN, Rizavi HS, Ren X, et al. Toll-like receptors in the depressed and suicide brain. J Psychiatr Res 2014;53:62-8. 
19. Schroder K, Tschopp J. The inflammasomes. Cell 2010;140:821-32.

20. Martinon F, Burns K, Tschopp J. The inflammasome: a molecular platform triggering activation of inflammatory caspases and processing of proIL-beta. Mol Cell 2002;10:417-26.

21. Agostini L, Martinon F, Burns K, et al. NALP3 forms an IL-1betaprocessing inflammasome with increased activity in Muckle-Wells autoinflammatory disorder. Immunity 2004;20:319-25.

22. Hanamsagar R, Torres V, Kielian T. Inflammasome activation and IL-1beta/IL-18 processing are influenced by distinct pathways in microglia. J Neurochem 2011;119:736-48.

23. First MB, Spitzer RL, Gibbon M, et al. Structured clinical interview for DSM-IV axis I disorders (SCID-I). Arlington (VA): American Psychiatric Publishing, Inc.; 1997.

24. Lowry OH, Rosebrough NJ, Farr AL, et al. Protein measurement with the Folin phenol reagent. J Biol Chem 1951;193:265-75.

25. Pandey GN, Dwivedi Y, SridharaRao J, et al. Protein kinase C and phospholipase $C$ activity and expression of their specific isozymes is decreased and expression of MARCKS is increased in platelets of bipolar but not in unipolar patients. Neuropsychopharmacology 2002;26:216-28.

26. Gillespie MT, Horwood NJ. Interleukin-18: perspectives on the newest interleukin. Cytokine Growth Factor Rev 1998;9:109-16.

27. Anderson JP, Mueller JL, Rosengren S, et al. Structural, expression, and evolutionary analysis of mouse CIAS1. Gene 2004;338:25-34.

28. Swanson KV, Deng M, Ting JP. The NLRP3 inflammasome: molecular activation and regulation to therapeutics. Nat Rev Immunol 2019;19:477-89.

29. Zhou K, Shi L, Wang Y, et al. Recent advances of the NLRP3 inflammasome in central nervous system disorders. I Immunol Res 2016;2016:9238290.

30. Schroder K, Zhou R, Tschopp J. The NLRP3 inflammasome: a sensor for metabolic danger? Science 2010;327:296-300.

31. Song L, Pei L, Yao S, et al. NLRP3 inflammasome in neurological diseases, from functions to therapies. Front Cell Neurosci 2017;11:63.

32. Glass CK, Saijo K, Winner B, et al. Mechanisms underlying inflammation in neurodegeneration. Cell 2010;140:918-34.

33. de Rivero Vaccari JP, Dietrich WD, Keane RW. Activation and regulation of cellular inflammasomes: gaps in our knowledge for central nervous system injury. J Cereb Blood Flow Metab 2014;34: 369-75.

34. Kim HK, Andreazza AC, Elmi N, et al. Nod-like receptor pyrin containing 3 (NLRP3) in the post-mortem frontal cortex from patients with bipolar disorder: a potential mediator between mitochondria and immune-activation. J Psychiatr Res 2016;72: 43-50.

35. Alcocer-Gomez E, de Miguel M, Casas-Barquero N, et al. NLRP3 inflammasome is activated in mononuclear blood cells from patients with major depressive disorder. Brain Behav Immun 2014; 36:111-7.

36. Syed SA, Beurel E, Loewenstein DA, et al. Defective inflammatory pathways in never-treated depressed patients are associated with poor treatment response. Neuron 2018;99:914-924, e913.

37. Alcocer-Gomez E, Ulecia-Moron C, Marin-Aguilar F, et al. Stressinduced depressive behaviors require a functional NLRP3 inflammasome. Mol Neurobiol 2016;53:4874-82.

38. Pan Y, Chen XY, Zhang QY, et al. Microglial NLRP3 inflammasome activation mediates IL-1beta-related inflammation in prefrontal cortex of depressive rats. Brain Behav Immun 2014;41:90-100.

39. Shi H, Wang Y, Li X, et al. NLRP3 activation and mitosis are mutually exclusive events coordinated by NEK7, a new inflammasome component. Nat Immunol 2016;17:250-8.
40. Vanaja SK, Rathinam VA, Fitzgerald KA. Mechanisms of inflammasome activation: recent advances and novel insights. Trends Cell Biol 2015;25:308-15.

41. Bauernfeind FG, Horvath G, Stutz A, et al. Cutting edge: NF-kappaB activating pattern recognition and cytokine receptors license NLRP3 inflammasome activation by regulating NLRP3 expression. I Immunol 2009;183:787-91.

42. Zambetti LP, Laudisi F, Licandro G, et al. The rhapsody of NLRPs: master players of inflammation ... and a lot more. Immunol Res 2012;53:78-90.

43. Meng G, Zhang F, Fuss I, et al. A mutation in the Nlrp3 gene causing inflammasome hyperactivation potentiates Th17 cell-dominant immune responses. Immunity 2009;30:860-74.

44. Mariathasan S, Newton K, Monack DM, et al. Differential activation of the inflammasome by caspase- 1 adaptors ASC and Ipaf. Nature 2004:430:213-8.

45. Iyer SS, He Q, Janczy JR, et al. Mitochondrial cardiolipin is required for Nlrp3 inflammasome activation. Immunity 2013;39 311-23.

46. Zhou R, Yazdi AS, Menu P, et al. A role for mitochondria in NLRP3 inflammasome activation. Nature 2011;469:221-5.

47. Franchi L, Park JH, Shaw MH, et al. Intracellular NOD-like receptors in innate immunity, infection and disease. Cell Microbiol 2008;10:1-8.

48. Gasse P, Riteau N, Charron S, et al. Uric acid is a danger signal activating NALP3 inflammasome in lung injury inflammation and fibrosis. Am J Respir Crit Care Med 2009;179:903-13.

49. Griffith JW, Sun T, McIntosh MT, et al. Pure hemozoin is inflammatory in vivo and activates the NALP3 inflammasome via release of uric acid. J Immunol 2009;183:5208-20.

50. Martinon F, Petrilli V, Mayor A, et al. Gout-associated uric acid crystals activate the NALP3 inflammasome. Nature 2006;440:237-41.

51. Nakahira K, Haspel JA, Rathinam VA, et al. Autophagy proteins regulate innate immune responses by inhibiting the release of mitochondrial DNA mediated by the NALP3 inflammasome. Nat Immunol 2011;12:222-30.

52. Andreazza AC, Shao L, Wang JF, et al. Mitochondrial complex I activity and oxidative damage to mitochondrial proteins in the prefrontal cortex of patients with bipolar disorder. Arch Gen Psychiatry 2010;67:360-8.

53. Iwata M, Ota KT, Duman RS. The inflammasome: pathways linking psychological stress, depression, and systemic illnesses. Brain Behav Immun 2013;31:105-14.

54. McAfoose J, Baune BT. Evidence for a cytokine model of cognitive function. Neurosci Biobehav Rev 2009;33:355-66.

55. McAfoose J, Koerner H, Baune BT. The effects of TNF deficiency on age-related cognitive performance. Psychoneuroendocrinology 2009;34:615-9.

56. Heneka MT, Kummer MP, Stutz A, et al. NLRP3 is activated in Alzheimer's disease and contributes to pathology in APP/PS1 mice. Nature 2013;493:674-8.

57. Bergsbaken T, Fink SL, Cookson BT. Pyroptosis: host cell death and inflammation. Nat Rev Microbiol 2009;7:99-109.

58. Suzuki T, Franchi L, Toma C, et al. Differential regulation of caspase- 1 activation, pyroptosis, and autophagy via Ipaf and ASC in Shigella-infected macrophages. PLoS Pathog 2007;3:e111.

59. Ojala J, Alafuzoff I, Herukka SK, et al. Expression of interleukin-18 is increased in the brains of Alzheimer's disease patients. Neurobiol Aging 2009;30:198-209.

60. White CS, Lawrence CB, Brough D, et al. Inflammasomes as therapeutic targets for Alzheimer's disease. Brain Pathol 2017;27:223-34. 